\title{
NORMALIZED NUMERICAL RANGES OF SOME OPERATORS
}

\author{
L. Z. GEVORGYAN
}

\begin{abstract}
We describe the normalized numerical ranges of certain operators. First, the case of a normal operator, acting in a two dimensional space is considered in detail, leading to the general Kantorovich inequality. Then we pass to the finite dimensional case and settle the problem when the Gustafson-Seddighin two component property, failing in general, takes place. In the next part finite and infinite dimensional Jordan cells are investigated.We conclude with the description of the normalized numerical range of a two-dimensional Toeplitz matrix.
\end{abstract}

Mathematics subject classification (2000): 47A12, 47B15, 47N40.

Keywords and phrases: normalized numerical range, convergence rate, Kantorovich inequality.

\section{REFERENCES}

[1] W. AUZINGER, Sectorial operators and normalized numerical range, Appl. Numer. Math. 45 (2003), 367-388.

[2] L. GEVORGYAN, On the convergence rate of iterations and the normalized numerical range, Math. Sci. Res. J. 8 (2004), no. 1, 16-26.

[3] L. Gevorgyan, On some ill conditioned operator equations, Dokl. Nats Akad Nauk Armen. 107 (2007), no. $2,111-117$.

[4] K. GUSTAFSON, D. RaO, Numerical Range, Springer, Berlin, 1997.

[5] L. V. KANTOROVICH, Funkcional'ny analiz i prikladnaya matematika, Uspehi Mat Nauk. 3(28), 89-185 (1948).

[6] M. SedDighin, Antieigenvalues and total antieigenvalues of normal operators, J. Math. Anal. Appl. 274 (2002) 239-254. 